\title{
The temperature reduction of components in the induction hobs of domestic kitchens by numerical and visualization models
}

\author{
E. Castillo ${ }^{1}$, M. Miana ${ }^{1}$, L. Sanagustín ${ }^{1}$, I. Hupont ${ }^{1}$, \\ M. Valencia ${ }^{2}$ \& S. Aranda ${ }^{2}$ \\ ${ }^{1}$ Instituto Tecnológico de Aragón, Spain \\ ${ }^{2}$ BSH Electrodomésticos España, Spain
}

\begin{abstract}
Significant heat flows are generated inside of induction hobs of domestic kitchens, so they must be dissipated without large rises of temperature to avoid thermal damage in these systems. However, the compact design of these devices impedes effective dissipation techniques like fins or fans near to the heating sources. Besides, the complex shapes of the equipment and the large number of computational cells involved in the numerical model requires advances in visualization techniques to make the understanding of the simulation results easier. In this study, the thermal behaviour of the inductor hob is represented by a detailed CFD model, which is validated against experimental measurements. The analysis of the temperature fields and the exchanged heat flows helps to propose new designs for reducing the temperature, specifically, for the plastic components, which are mainly heated by the copper coils and cooled by the ferrites and the aluminum tray. Thus, it is proposed that the elimination of the plastic grooves that surround the copper spirals and the increment of the thermal conductivity of the material that connects plastic and ferrites. In both cases, the maximum temperature is reduced. For visualization purposes, a textured low poly $3 \mathrm{D}$ model is built from the high poly colored mesh resulting from full numerical CFD calculations. This solution allows the numerical results of this system in both PC and mobile devices to be shown.
\end{abstract}

Keywords: induction hob, conjugate heat transfer, CFD, interactive visualization techniques. 


\section{Introduction}

Some types of materials, when are subjected to electromagnetic fields, absorb part of its energy and transform it into heat. These materials are called ferromagnetic materials, usually metals with high electrical conductivity [1]. Several works on the literature develop different techniques to improve induction heating on commercial devices. These improvements are focused on quick heating up, accurate temperature control [2], inductors with adaptable diameter [3] or changes on the vessel properties [4], regardless on the heat fluxes which are transferred in the inner parts of the system. On the other hand, the inner electronic components are the principal-non desired heat sources of industrial devices, and the heat transferred from them is dramatically relevant for the correct behaviour of the device. In order to dissipate this thermal energy, some works are devoted to define multiple facets of systems which are dependent on convective thermal losses [5], while some others are focused on the improvements of the system thermal operation, for example by means of the optimization of the geometrical aspects in canonical systems [6] or by optimization of the boundary conditions of the system [7].

The main objective of this investigation is the reduction of the temperature of the elements that compose the induction hobs of domestic kitchens, by means of intensive numerical simulations in steady state considering two recipients at two different power supplies. This target has two important advantages: first, the reduction of the temperatures of components moves away their risk of thermal damage. Second, the ferromagnetic properties of some materials used at induction hobs show significant variation with temperature, and their values at large temperature are poor. A detailed control of temperatures in the ferrites, for example, will improve their isolating magnetic effects in the rest of system. Due to the large number of bodies that compose the induction hobs, the current paper investigates only the temperature reduction in the plastic components, where the heat dissipation is quite complex. This analysis allows a detailed description of the temperature each part that composes the induction hob and the heat transferred between them. Experimental measurements of temperature are performed to validate this model. The computational analysis makes easier the detection of hot spots in plastic bodies that can trouble the device operation. Once the system behavior is well known, two design modifications are proposed and numerically tested to reduce the average and the maximum temperature of the plastic pieces. Besides, the current investigation searches for an efficient way to visualize the obtained computational data. Intensive numerical simulations, such as the considered in this work, typically make use of high order data, and cause significant obstacles when attempting to generate accurate visualizations. The fact of handling meshes with a large number of polygons implies too much computational time and storage to obtain a single rendered image or animation from the $3 \mathrm{D}$ model, and consequently real time interaction with the model is not viable. Although yet unexplored in the literature [8] a real time, advanced and interactive way of visualizing the $3 \mathrm{D}$ results from computational fluid dynamics simulations provide a high added value to this kind of studies, and is performed 
here. This paper is composed of six sections. This first one introduces the general framework of the investigation, the second one depicts the analyzed system and the experimental procedure. Section 3 describes the numerical model and the validation, and section 4 analyses the obtained results and proposes two modifications. The visualization procedure is described in section 5 and finally, section 6 summarizes the main conclusions for this research.

\section{Experimental tests}

The system is composed by four burners of about $200 \mathrm{~mm}$ of diameter. A glass is placed over these components, and one of the two selected objects to be heated is put on it. The first one is a disk of the same diameter as the induction and $6 \mathrm{~mm}$ of height, and the second one is the same disk, but attached to an aluminum dissipater composed of 19 fins. There is a fan attached at one side of the dissipater which impulses an unknown air flow rate through the fin channels to improve the heat transfer from these surfaces. The inductors are placed just under the glass, separated only by a slim disk of mica. Each inductor is formed by a plastic frame with several grooves, where the copper coil has been inserted, forming a spiral and ferrites have been stuck with tape under the plastic frame. There are two aluminum trays which support a pair of inductors to puss them to the contact with the glass by the mica disks, and three springs push each tray and the inductors against the glass. Under the aluminum trays, there is a fan, a radiator to cool the IGBT devices and a set of electronic components. Inductor $\mathrm{n}$. 1 is just switched on, which is the rear one over the right tray according to the inlet flow driven by the fan. Only the electronic components of a characteristic size larger of $5 \mathrm{~mm}$ have been included in the geometry to be meshed. Experimental measurements are performed under EN 60335-2-6 specifications [9] to validate the computational model and to obtain initial description of the thermal behavior of the system. Up to $26 \mathrm{~K}$ type thermocouples are located inside of the induction hob, 3 thermal probes over the disk surface and $7 \mathrm{~K}$ type thermocouples over the dissipater. The thermocouples are mainly placed around the switched inductor. The channels 1 to 4 are located inside of the plastic, in the symmetry plane of a ferrite, while the channels 8 to 11 are placed on a plastic nerve far away from the ferrite. These two locations are kept for the thermocouples 5 to 7 and 12 to 14, placed over the aluminum tray but near or not to the ferrite. The temperature of the mica disk on inductor $\mathrm{n}$. 1 is obtained by thermocouples in the channels 15 to 19 , while the channels 20 to 22 are specifically devoted to measure the temperature in the ferrites. The fluid temperature is measured by the channel 23 under the aluminum tray while the glass temperature is recorded by thermocouples in channels 24 to 26 . The thermal probes on the disk surface are stored by the channels 27 to 29 , and the channels t 1 to $t 7$ are set up on different parts of the heat dissipater. Four experimental tests get the steady temperature in these locations, depending on the heat generation. For the disk, $90 \mathrm{~W}$ (D90) and $180 \mathrm{~W}$ (D180) are imposed, and for the dissipater $850 \mathrm{~W}$ (HD850) and $1050 \mathrm{~W}$ (HD1050). The real system and the simulated domain is shown in fig. 1. Both experimental sets start from 
the ambience temperature, and the power is still switched on for a certain period of at least $2 \mathrm{~h}$, to ensure the achievement of steady state conditions. After that, the electric power is disconnected and the system is allowed to return to the initial state. This investigation analyses only the temperature at the steady state, and the results are shown in non-dimensional formulae, due to the confidential nature of the investigation.
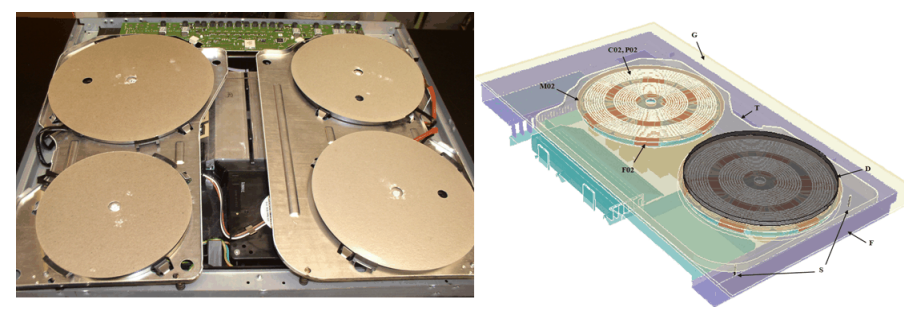

Figure 1: Right: real system, without the upper glass. Left: simulated domain (half of the real system) for the disk case.

\section{Computational models}

\subsection{Governing equations and numerical methods}

The problem to be solved can be defined by the Navier-Stokes equations for the fluid flow, assuming the hypothesis of Newtonian fluid and incompressible steady flow. The solid domain is defined by the energy transport equation considering conduction and volumetric heat generation owing to induction phenomena. Transport equations are solved by the Finite Volume Method using ANSYS-FLUENT software, release 14.0 [10] and double precision is used for the pressure-based solver. The convergence is improved by the Algebraic Multigrid Scheme [11], and second order spatial discretisation is used for the solved variables. The SIMPLE algorithm [12] is selected for solving the pressure velocity coupling. When the fan is turned on, turbulence in fluid flow and in heat transfer is modeled by k- $\varepsilon$ standard model [13] with standard wall functions [14]. For the cases in which the fan is turned off, the flow is assumed to be laminar. The numerical domain is first simplified to the half of the experimental system for symmetry reason. Thus, the numerical model is composed only of 2 inductors of about $200 \mathrm{~mm}$ placed over an aluminum tray. The initial tetrahedral mesh is reduced to a polyhedral mesh, composed of 5.05 million cells.

\subsection{Materials and heat generation}

Thermal properties of the solid materials are listed in table 1. The tray, the dissipater and the interior frame are composed of aluminum; steel is applied at the disk and tape is the material used to join the ferrites to the plastic. The air density is calculated from the incompressible ideal gas state equation, and viscosity, heat capacity and thermal conductivity are linearly interpolated with temperature from tabulated values from Incropera and De Witt [15]. 
Table 1: Thermal properties of solid materials.

\begin{tabular}{|l|l|l|l|l|l|l|l|l|}
\hline & Steel & Aluminum & Copper & Glass & Ferrite & Mica & Plastic & Tape \\
\hline$\rho(\mathrm{kg} / \mathrm{m} 3)$ & 7820 & 2730 & 8930 & 2500 & 480 & 2800 & 1950 & 2730 \\
\hline $\mathrm{CP}(\mathrm{J} / \mathrm{kgK})$ & 500 & 893 & 385 & 800 & 800 & 836 & 860 & 893 \\
\hline$(\mathrm{W} / \mathrm{mK})$ & 50.8 & 154 & 398 & 1.7 & 3 & 0.8 & 0.58 & 0.06 \\
\hline
\end{tabular}

The electronic power loss is not directly applied into the heat generating bodies. First, there is a process efficiency, ranging from 92 to $95 \%$, that removes the power dissipated by electronic components such as IGBTs. The remainder of the total power supply is next split into the induced power on the container and the volumetric power losses in solids. The power losses in the aluminum tray are limited to a two concentric rings under the induction hob, and the induced power at the container base follows the classical radial profile starting and ending in 0 and having two maximums in the mid zone [4].

\subsection{Boundary conditions}

Four different types of boundary conditions are applied in the numerical model: a flow inlet, a flow outlet, walls and interfaces. At the inlet, the temperature is set to $318 \mathrm{~K}$, which is the air enclosed temperature by the kitchen furniture at steady state. For the dissipater, the fan is switched on, so the flow inlet is defined by a uniform velocity profile of $1.98 \mathrm{~m} / \mathrm{s}$ to yield the target mass flow rate. In these cases, the flow is considered turbulent, with a turbulent intensity of $5 \%$ and an hydraulic diameter $0.01 \mathrm{~m}$. When the fan is switched off, the flow is laminar and the fluid variables are calculated by imposing a total gauge pressure of $0 \mathrm{~Pa}$. In both situations, when the fan is either on or off, the flow variables at the outlet are fixed by imposing a static gauge pressure of $0 \mathrm{~Pa}$.

At wall zones, momentum equations are solved by imposing the non slip condition. The walls of the electronic components, the radiator and at all the supporting devices walls are considered adiabatic. A convective heat transfer coefficient and an emissivity are imposed at the external walls of the simulated domain to allow the heat exchange with the environment. The glass and the container walls transfer heat to the environment at $300 \mathrm{~K}$, and emissivity is set to 0.8 for dark walls (those from plastic, glass or disk) and 0.1 for polished surface of the interior frame and fin dissipater.

A major feature of this numerical model is the use of non conformal meshes, where the node locations are not the same at both sides of some cell zones, to join some solid and fluid zones without a prohibitive increment in the number of cells. The connections of different meshes are performed by interface boundary conditions [10]. However it has a major drawback: the number of boundary conditions is highly enlarged because the connection between two different volumes by means of interfaces can generate up to 4 new boundary conditions. The numerical model presented is defined by more than 800 boundary conditions and its post-processing must be performed by automatic procedures. 


\subsection{Validation}

To validate the numerical model, the temperature predicted by computational simulations is compared in fig. 2 with the steady experimental data obtained in section 2. The comparison is made easier by displaying the non dimensional difference between experimental and numerical temperatures defined in eqn (1).

$$
\Delta=\frac{T_{C F D}-T_{E x p}}{T_{E x p}}
$$

It is observed a good agreement in channels 1 to 4 and 8 to 11 (the inductor plastic), where the relative difference is below $10 \%$ in all cases. Channels 6 and 7 (the inductor plastic, close to the ferrite) are in good agreement except in the disk cases, but the maximum difference is about $17 \%$, yielding reasonable accuracy of the predicted values. In channels 17 to 19 (mica zone), where the differences are lower than $10 \%$ but in the disk cases, the largest difference reached is $20 \%$ for the smallest power. These differences can be provoked by the uniform heat generation imposed at the solids. Channel 12 (joint of the inductor and the aluminum tray), reaches a higher temperature in the experimental data. Nevertheless, channel 5, which is placed in the same zone, but closer to the ferrites, reaches a temperature lower than the experimental result in all cases but in disk $90 \mathrm{~W}$, probably caused by the uniform heat generation imposed. Channels 20 to 22 show an irregular behavior, likely due to the coupling of thermal and electromagnetic effects. Numerical results show a significant variation of temperature in vertical direction owing to the thermal resistance induced by the gap between the ferrites and the inductor plastic. The same behavior is observed in channel 23 , located at the fluid zone, at $8 \mathrm{~mm}$ under the aluminum tray. The temperature obtained from numerical simulations varies large in downward vertical direction: the evaluated value $2 \mathrm{~cm}$ downward is close to the experimental results, so the uncertainty in the right position of the thermal sensor can be considered as the responsible of these differences. Channels 24 to 26 are located over the glass and the differences reach values up to $25 \%$, justified by the neglected influence of the disk in the radiated heat transfer from the adjacent glass and in the constant heat transfer coefficient. The accuracy of the prediction of temperature in thermocouples $\mathrm{t} 1$ to $\mathrm{t} 7$ varies between $8 \%$ and $25 \%$ but it is not studied in detail, due to the rough estimation performed in the heat transfer coefficient. The numerical model is not able to reproduce the difference in thermocouples 27 to 29 (disk surface), and the reason probably lies in some magnetic induction effects which have not been taking into account in this simplified thermal model. Finally, the independency of numerical results with respect of the mesh size is ensured. Fig. 3 compares the values obtained for the D180 case from the selected mesh with the ones obtained from a coarse mesh with 4.08 million of cells and a fine mesh of 11.71 millions cells. The fine mesh yields no significant improvements to the accuracy of numerical results from the selected mesh but it increases twice the computational time, so it is discarded. The coarse mesh converge rate is quite poor, so it is discarded too. 


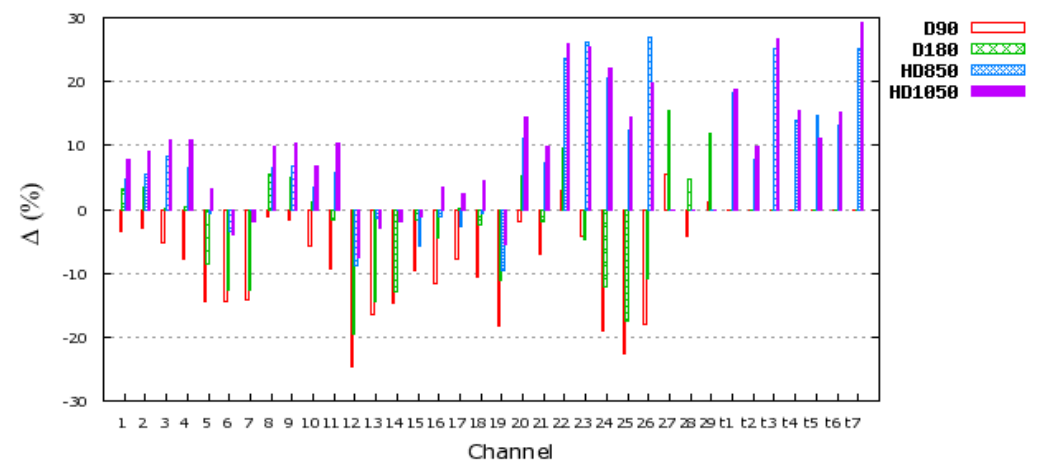

Figure 2: Difference between numerical and experimental temperatures.

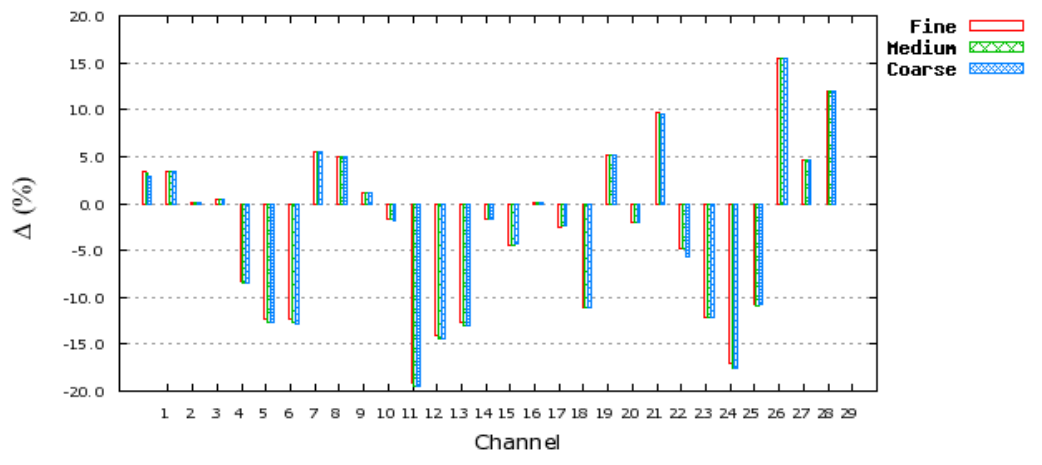

Figure 3: Influence of grid size for the D180 case.

\section{Results}

\subsection{Heat flux analysis}

The heat fluxes are presented on fig. 4, in terms of the non-dimensional heat flux $Q^{*}$ defined on eqn (2) and expressed in terms of $Q_{0}$ and $Q_{\text {Ref }}$, which are two arbitrary reference heat fluxes, equal for all the cases performed.

$$
Q^{*}=\frac{Q_{C F D}-Q_{\text {Ref }}}{Q_{0}-Q_{\text {Ref }}}
$$

On the horizontal axis, the abbreviated names of two solids are written and separated by a "-" sign. The first name is the solid from the heat flow starts, and the second name is the solid to the heat goes, and negative values means that heat goes in opposite direction. The abbreviations employed are " $\mathrm{A}$ " for the air placed above the tray, "C01-02" for the coppers of hob n. 1 and hob n. 2 respectively, "D" for the disk, "E" for the environment, "F" for the frame, "F01- 
02 " for the ferrites, "G" for the glass, "IA" for the inferior air, "M01-02" for the micas, "P01" and "P02" for the plastics, "S" for the springs located under the aluminum tray and " $\mathrm{T}$ " for the tray.

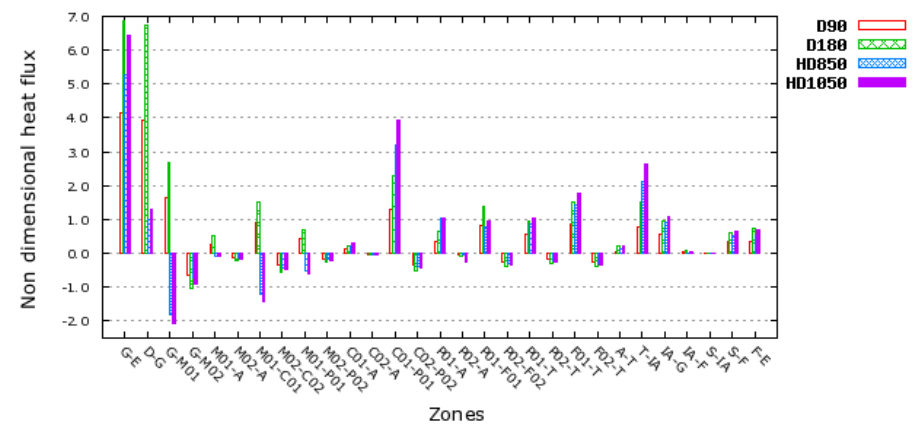

Figure 4: Exchanged heat fluxes.

The induced power generated in the base of the container is mainly dissipated by the container. However, there is a certain amount transferred to the glass, which is governed by the thermal resistance that characterizes the heat exchange between the container and the environment and by the temperature difference between the container and the solids inside of the induction hob. The disk has the largest thermal resistance to the heat transfer to the environment owing to its small heat transfer area and convective heat transfer coefficient. Thus, both disks cases have the biggest percentage of heat flux transferred to the glass compared to the heat generated at the base of the container. On the other hand, the aluminum dissipater presents a lower resistance, and it causes that the heat transferred to the glass is less. These differences in the heat transfer area and in the heat transfer coefficient from the container to the environment are the responsible for the larger allowable heat generation in the case of the dissipater compared to the disk. Copper coils in inductor n. 1 are the next body with a volumetric power loss generated inside it. For cases with high power supply, as in the heat dissipater tests, the generated heat is transferred mainly to the mica sheet and to the plastic frame, and the heat exchanged with the air is negligible. However, for the disks cases, the most of the heat is transferred to the plastic framework and the copper coils receive a significant heat flow from the mica disk, which is transferred to the plastic structure. The appropriate transmission of these heat flows to other parts different from plastic will probably yield a reduction of the temperature of it. There is a small volumetric power loss produced in aluminum tray, which additionally receives heat flow from the air enclosed by the tray itself, from the plastic frame and from ferrites of inductor $n$. 1. In contrast, the aluminum tray heats the ferrites and the plastic of inductor n. 2, the springs and the air volume located under it. The heat received by conduction from other bodies is larger than the heat generated inside of the tray. On inductor n. 2, the heat transferred from the aluminum tray to the plastic frame and to the ferrites, which are then transferred to the plastic structure, is quite 
similar for all the cases. The total heat received by the plastic body is next lead to copper coils and mica sheet with the same relation in all the cases, and the copper spirals obtain an additional heat flow from the air enclosed by the aluminum tray. Finally, the copper spirals of inductor $n .2$ heat the mica disk and the total heat flow received by mica disk from the plastic and the copper solids is dissipated to the environment through the glass.

\subsection{Design modifications}

The reduction of the temperature field of the plastic hob n. 1 is not straightforward, because is connected to the copper coils, the ferrites and the aluminum tray, which generate heat inside of them. Besides, depending on the object to be heated, there is a heat flow from the mica to the inductor. Moreover, convection does not play a significant role in the cooling of plastic bodies even when the fan is switched on. Design modifications search for efficient solutions to modify the largest heat flows which involve the plastic structure. On the first modification (MOD01), the plastic surrounding copper is partially eliminated, and there is only air between some parts of the copper spirals. Plastic volume on inductor decreases, plastic-copper contact surface is reduced, and copper-air contact is increased. The idea underlying this modification is the diverting of a part of the heat transferred from the copper coils to the air placed above the tray, reducing then the heat flow received by the plastic inductor from the copper coils. In contrast, second modification (MOD02) searches for the increment of the cooling heat flow from the plastic to the ferrites, by the dissipation through the aluminum tray to the inferior part of the system. Plastic and ferrites parts are stuck by a tape strip having a thermal conductivity of $0.06 \mathrm{~W} / \mathrm{mK}$. The tape is substituted by a metallic foil tape with a thermal conductivity of $5 \mathrm{~W} / \mathrm{mK}$. Results for the performed modifications over plastic frames are presented in table 3 , where the non-dimensional temperatures are calculated by the computational values divided by the heat deflection temperature $\left(\mathrm{T}_{\mathrm{HD}}\right)$ of the plastic material measured at $1.8 \mathrm{MPa}$ according to ASTM D648 norm [16].

The suppression of the plastic grooves in MOD01 reduces the maximum temperature of the plastic hob n. 1 less than $1 \%$ with respect to the values obtained for the initial design. Even, the maximum temperature is slightly augmented for the case of HD1050. However, the average temperature is reduced, less than $1 \%$ for all cases, and the standard deviation of temperature is increased by $7 \%$ with respect to the values obtained for the initial design. The changes of the standard deviation and average temperature together with the nearly constant values of the maximum temperature point that cooling of the plastic inductor is better with this new design, but its risk of thermal damage keeps practically constant. Regarding MOD02, the increment of the thermal conductivity of the tape reduces weakly the risk of thermal damage in all analyzed cases. For instance, the maximum temperature is reduced up to $1.16 \%$ with respect to the initial values obtained for the case with the highest risk of thermal damage, that is, the HD1050 case. In this case, both the average temperature and the standard deviation are too reduced. 
Table 2: Temperature results for plastic of inductor $n .1$.

\begin{tabular}{|c|c|c|c|c|}
\hline Design & Case & $T_{\text {Max }}{ }^{*}$ & $\mathrm{~T}_{\text {Ave }}{ }^{*}$ & $\sigma_{\mathrm{T}}$ \\
\hline Initial & \multirow{3}{*}{ D180 } & $85.019 \%$ & $74.776 \%$ & $7.693 \%$ \\
\hline MOD01 & & $85.003 \%$ & $74.245 \%$ & $8.249 \%$ \\
\hline MOD02 & & $84.457 \%$ & $74.530 \%$ & $7.261 \%$ \\
\hline Initial & \multirow{3}{*}{ HD1050 } & $90.283 \%$ & $79.449 \%$ & $8.848 \%$ \\
\hline MOD01 & & $90.402 \%$ & $79.071 \%$ & $9.433 \%$ \\
\hline MOD02 & & $89.236 \%$ & $79.099 \%$ & $8.492 \%$ \\
\hline Initial & \multirow{3}{*}{ D90 } & $55.390 \%$ & $49.476 \%$ & $4.444 \%$ \\
\hline MOD01 & & $55.231 \%$ & $49.038 \%$ & $4.762 \%$ \\
\hline MOD02 & & $54.642 \%$ & $48.676 \%$ & $4.147 \%$ \\
\hline Initial & \multirow{3}{*}{ HD850 } & $75.915 \%$ & $67.182 \%$ & $7.165 \%$ \\
\hline MOD01 & & $75.279 \%$ & $65.927 \%$ & $7.855 \%$ \\
\hline MOD02 & & $74.909 \%$ & $66.697 \%$ & $6.920 \%$ \\
\hline
\end{tabular}

\section{Visualization}

Solving of the transport equations for the fluid flow makes use of high poly 3D meshes, representing the object being studied to compute finite volume based CFD calculations. It offers visualization capabilities, however, since it handles meshes with a large number of polygons, a long computational time and storage is needed to obtain each rendered image or animation from the $3 \mathrm{D}$ model. Therefore real time interaction with the model is not viable and visualization possibilities are limited. This work aims to make the visualization of CFD simulations an advanced and useful interactive 3D experience. To achieve this goal, a post process of the high poly CFD results is needed in order to obtain a low poly mesh of the system to be handled in real time by a 3D engine. In order to avoid losing precision in the visualization, the high order computed elements must be preserved at their original locations, even though the $3 \mathrm{D}$ model has a lower number of vertices. The process shown in fig. 5 has been carried out for each piece, composing the system 3D model. Firstly, the high poly data that serves as an input to the CFD analysis is obtained by meshing the original induction hob model provided by the manufacturer. As a result of the CFD computation process, a file in standard PLY format [17] is built from the data: it contains the spatial coordinates of each vertex, followed by the RGB values codifying the color of the temperature computed at this specific location. With this information, and by means of the render to texture technique [18], a bitmap texture is extracted for each piece, which is then applied to the original low poly mesh, and the final post processed induction hob 3D model has a low number of polygons, and therefore is suitable to be used in real time by any graphic engine, without losing any data calculated from the high poly model. In order to show the potential of the post processing methodology, an interactive application has been created with the UNITY multiplatform game engine [19] and compiled for Windows PC, Android and iOS operating systems. The application allows the user to assemble, disassemble and explore the different pieces of the induction hob model by interacting with the mouse or through a tactile interface. The multiplatform deployment capabilities offered by Unity open the door to reuse 
the same code in a future to deploy the application in other platforms, such as CAVE like environments, game consoles or web pages. Finally, an example for the applied technique which shows the CFD temperature results obtained is presented in fig. 5. The temperature scale is not numerically shown, due to the confidential nature of the results.
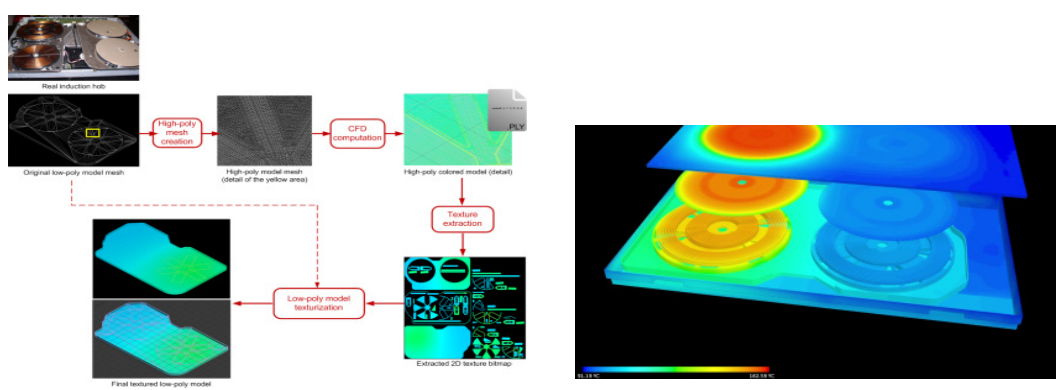

Figure 5: Right: Textured low poly model for real time interactive visualization scheme. Left: example of temperature results.

\section{Conclusions}

A numerical model is developed to predict the thermal behavior of an induction hob. This simulation model is validated through four tests, combining different heat sinks and supplied power levels yielding diverse working temperatures. The difference between numerical and experimental temperatures is lower than $10 \%$ in all cases for 8 channels located over the inductor. This difference is enlarged for the channel that measure the fluid temperature under the aluminum tray and the channels located inside of ferrites, owing to the uncertainties in the exact location of thermocouples and the electromagnetic and thermal coupling effects in ferrites. Once the numerical model is validated, the thermal behavior of the system is described by means of the heat transfer in the system and two modifications to the initial design of inductor n. 1 are proposed. First option reduces the contact surface of the plastic part with the copper coils, but this design yields negative results for the maximum temperature in the case of the largest power supply, although it achieves the proposed objective for the rest of analyzed variables and cases. The second design increases the thermal conductivity of the tape that sticks the plastic hob and ferrites, and gets a weak reduction of temperature in all analyzed cases so it can be considered as a first step towards major improvements in future researches. This paper has also proposed a method to build a textured low poly 3D model from the high poly colored mesh resulting from CFD calculations for visualization purposes. The presented technique is accurate, since it preserves the high level data solutions at their original locations, and novel given that the fact of using low poly meshes allows real time interactive multiplatform visualization. Thanks to the UNITY game engine, the interactive experience has been made possible even in devices with low computational power, such as iOS and Android smart-phones or tablets. 


\section{References}

[1] Rudnev V., Loveless D., Cook R, Black M. Handbook of Induction Heating, Marcel Dekker: New York, 2003.

[2] Paesa D., Franco C., Llorente S., Lopez, Nicolas G., Sagües C., Adaptive Simmering Control for Domestic Induction Cooker. IEEE Trans. Ind. Appl., 47(5), pp. 2257-2267, 2011.

[3] Carretero C., Millán I, Lucía O., Alonso R., Burdío J.M., Analysis and Modeling of Planar Concentric Windings Forming Adaptable-Diameter Burners for Induction Heating Appliances. IEEE Trans. Power Electron, 26(5), pp. 1549-1558, 2011.

[4] Koller L. \& Novák B., Improving the Energy Efficiency of Induction Cooking. Electrical Engineering, 91(3), pp. 153-160, 2009.

[5] Hung, T.C. \& Fu, C.S. Conjugate heat transfer analysis for the passive enhancement of electronic cooling through geometric modification in a mixed convection domain. Numer. Heat Tr. A-Appl. 35(5), pp. 519-535, 1999.

[6] Hsu, T.H. \& Wang, S.G. Mixed convection in a rectangular enclosure with discrete heat sources. Numer. Heat Tr. A-Appl. 38(6), pp. 627-652, 2000.

[7] Incropera, F.P., Kerby, J.S., Moffatt, D.F., Ramadhyani, S., Convection heat-transfer from discrete heat-sources in a rectangular channel J. Heat Mass Transfer, 29(7), pp. 1051-1058, 1986.

[8] Nelson B., Liu E., Kirby R.M., Haimes R., A System for the Accurate and Interactive Visualization of High-Order Finite Element Solutions. IEEE Trans. Vis. Comput. Graphics, 18(12), pp. 2325-2334, 2012.

[9] European Norm EN 60335-2-6:2003/A11 (2010).

[10] ANSYS-FLUENT Inc, (2011), ANSYS-FLUENT Theory Guide, Release 14.0.

[11] Hutchinson R. \& Raithby G.D., A Multigrid Method Based on the Additive Correction Strategy. Num. Heat Transfer, 9, pp. 511-537, 1986.

[12] Patankar S.V. and Spalding D.B., A Calculation Procedure for Heat, Mass and Momentum Transfer in Three-Dimensional Parabolic flows. Int. J. Heat Mass Transfer, 15, pp. 1787-1972, 1972.

[13] Launder B.E. and Spalding D.B., Lectures in Mathematical Models of Turbulence. Academic Press: London, 1972.

[14] Launder B.E. \& Spalding D.B., The Numerical Computation of Turbulent Flows. COMPUT METHOD APPL M, 3, pp. 269-289, 1974.

[15] Incropera F.P. and De Witt D. Fundamentals of Heat and Mass Transfer, John Wiley: New York, 1990.

[16] ASTM D648-07, Standard Test Method for deflection Temperature of Plastic Under Flexural Load in the Edgewise Position. www.astm.org

[17] PLY - Polygon File Format. http://graphics.stanford.edu

[18] Wynn, C, (2002), “OpenGL Render-to-Texture”. https://developer.nvidia.com

[19] UNITY, http://unity3d.com/unity/ 